\title{
Light-hole transitions in quantum dots: Realizing full control by highly focused optical-vortex beams
}

\author{
G. F. Quinteiro ${ }^{1,2, *}$ and T. Kuhn ${ }^{2}$ \\ ${ }^{1}$ Departamento de Física and IFIBA, FCEN, Universidad de Buenos Aires, Ciudad Universitaria, Pabellón I, \\ 1428 Ciudad de Buenos Aires, Argentina \\ ${ }^{2}$ Institut für Festkörpertheorie, Universität Münster, Wilhelm-Klemm-Straße 10, 48149 Münster, Germany
}

(Received 14 March 2014; revised manuscript received 19 August 2014; published 2 September 2014)

\begin{abstract}
An optical vortex is an inhomogeneous light beam having a phase singularity at its axis, where the intensity of the electric and/or magnetic field may vanish. Already well studied are the paraxial beams, which may carry well-defined values of spin (polarization $\sigma$ ) and orbital angular momenta; the orbital angular momentum per photon is given by the topological charge $\ell$ times the Planck constant. Here we study the light hole-to-conduction band transitions in a semiconductor quantum dot induced by a highly focused beam originating from a $\ell=1$ paraxial optical vortex. We find that at normal incidence the pulse will produce two distinct types of electron-hole pairs, depending on the relative signs of $\sigma$ and $\ell$. When $\operatorname{sgn}(\sigma)=\operatorname{sgn}(\ell)$, the pulse will create electron-hole pairs with band + spin and envelope angular momenta both equal to 1 . In contrast, for $\operatorname{sgn}(\sigma) \neq \operatorname{sgn}(\ell)$, the electron-hole pairs will have neither band+spin nor envelope angular momenta. A tightly focused optical-vortex beam thus makes possible the creation of pairs that cannot be produced with plane waves at normal incidence. With the addition of co-propagating plane waves or switching techniques to change the charge $\ell$ both the band + spin and the envelope angular momenta of the pair wave function can be precisely controlled. We discuss possible applications in the field of spintronics that open up.
\end{abstract}

DOI: 10.1103/PhysRevB.90.115401

PACS number(s): 42.50.Tx, 78.67.-n

\section{INTRODUCTION}

Quantum dots (QDs) are artificial systems that confine electrons and/or holes in all directions, yielding a discrete spectrum of energy levels. A variety of QDs have been fabricated and studied, such as laterally confined QDs in a two-dimensional electron gas, colloidal semiconductor nanocrystals, and semiconductor self-assembled QDs, to name only few. Due to their semiconductor nature, self-assembled QDs have electron energy levels grouped into valence (with heavy- and light-hole subbands) and conduction bands. In most cases, the uppermost energy levels in the valence band are of heavy-hole $(\mathrm{HH})$ type. The energy difference between bands makes it possible to excite electrons from the valence to the conduction band by optical or near-optical fields, and the selection rules of the optical transitions can be used to control the spin states of the generated electron-hole pairs. For this reason, applications to spintronics typically focus on the fast control of the spin state of carriers or nearby impurities by short-pulse excitation of electronic states in ensembles of QDs, and more recently in single QDs [1-4]. The studies mostly consider excitation from $\mathrm{HH}$ states, since decaying is reduced by the lack of lower energy levels. The recent literature shows an increasing interest in light-hole (LH) state excitation and manipulation [5-7]. A strong advantage of LHs over $\mathrm{HHs}$ is that, with their spin projections $\pm 1 / 2$, spin flips with, e.g., an impurity spin can occur [6]. However, the fast-decay channel leading to the lower HH levels makes it difficult to use LHs in proposals designed for the former. Recently Huo et al. [8] demonstrated energy inversion between hole subbands in stressed GaAs QDs. Their successful experiment widens the applicability of LHs to spintronics.

Strongly varying electromagnetic fields attract the attention of researchers in diverse areas such as optics, atomic and

*gquinteiro@df.uba.ar molecular physics, and information technology [9]. A prominent example is that of optical-vortex fields, characterized by a phase singularity where the intensity of the electric and/or magnetic field may vanish. Instances of beams with optical vortices are the radially/azimuthally polarized beams and twisted-light (TL) Bessel and Laguerre-Gaussian beams. It has been shown that a paraxial optical vortex carries orbital angular momentum (AM) equal to $\hbar \ell$, where $\ell$ is the topological charge. However, when such a beam is tightly focused, orbital and spin AM are not well separated [10] and the beam becomes a superposition of modes with different topological charge [11]. In solid-state systems, it has been shown that paraxial beams of TL bring about new effects such as circular photondrag effect in bulk [12] and nanostructures [13,14], new optical transitions in quantum dots [15], excitation of new exciton states [16-18], and excitation of intersubband transitions at normal incidence in quantum wells [19]. A number of authors have called attention to a special set of nonparaxial optical-vortex fields. They have shown that the values $\ell=1,2$ (to be understood as the value of the corresponding paraxial original beam) present especially interesting features [20-23]. For instance, close to the phase singularity and for circular polarization $\sigma=-1$ (polarization vector $\hat{x}-i \hat{y}$ ) and $\ell=2$ the magnetic interaction overcomes the electric interaction $[21,23,24]$. Or for the set $\{\ell=+1, \sigma=-1\}$ a nonvanishing longitudinal component of the electromagnetic field exists even at the phase singularity.

In this article we study the excitation of LHs in semiconductor QDs by pulses of highly focused beams of optical vortices with emphasis on the value $\ell=1$. We show that by only changing the polarization state of the light, electron-hole (e-h) pair states with and without band+spin and envelope AM can be addressed at normal incidence. Moreover, in conjunction with a co-propagating field with topological charge $\ell=0$, all states formed out of envelope $s$ and $p$ shells can be controlled. Compared to proposals using two orthogonally propagating 
pulses of plane waves, our normal-incidence configuration may significantly improve the experimental realization of $\mathrm{LH}$ state control in a single QD.

The article is organized as follows. Sections II and III introduce the material system and electromagnetic fields, respectively. The optical transitions are discussed in Sec. IV. Possible applications to spintronics are given in Sec. V. Section VI discusses some issues related to sample structures and experimental realizations of our proposed schemes. The article ends with the conclusions in Sec. VII.

\section{MATERIAL SYSTEM}

We will study the interaction of optical-vortex light with a single semiconductor self-assembled QD. In self-assembled QDs electron and hole states are confined on a nanometer scale. For small QDs the energy spectrum is a collection of discrete states grouped in two valence ( $\mathrm{LH}$ and $\mathrm{HH}$ ) bands and one conduction band. The valence bands are characterized by a total angular momentum $j=3 / 2$. In the $\mathrm{HH}$ band the $z$ components are given by $m_{j}= \pm 3 / 2$ while in the $\mathrm{LH}$ bands they are $m_{j}= \pm 1 / 2$. Here we neglect possible band mixing effects caused by strain distributions or the Luttinger Hamiltonian. In the conduction band the quantum numbers are $j=s=1 / 2$ and $m_{s}= \pm 1 / 2$. Conduction and valence bands are separated by a band-gap energy $E_{g}$ in the few $\mathrm{eV}$ range; thus, interband transitions may be produced by optical or near-optical excitation.

In general, the uppermost energy levels in the valence band are of $\mathrm{HH}$ type; however, an inversion between $\mathrm{LH}$ and $\mathrm{HH}$ bands is possible by applying stress to the sample [8]. The inversion of energy levels is advantageous in proposals using LH excitations, since it slows the decay of the states.

QDs can be fabricated out of different materials. Typical ones are InAs/GaAs or CdTe/ZnTe QDs, but others may prove to be more suitable in certain situations. In particular, QDs made of GaN/AIN $[25,26]$ have a large $E_{g}$ and therefore are excited at shorter wavelength. We will later discuss the possible advantage of these QDs.

We choose the $z$ axis of our cylindrical coordinate system collinear to the QD's growth direction; the QD has the shape of a disk with radius $r_{0} \simeq 10 \mathrm{~nm}$ and height $z_{0} \simeq 0.1 r_{0}$ and is centered at $r=0$. The complete wave function for carriers in the valence and conduction bands is

$$
\psi(\mathbf{r})=\phi(r, \varphi) \mathcal{Z}(z) u_{i}(\mathbf{r}),
$$

where the envelope functions are $\mathcal{Z}(z), \quad \phi_{i s n}(r, \varphi)=$ $\mathcal{R}_{i s n}\left(r / \ell_{c}\right)(2 \pi)^{-1 / 2} e^{i n \varphi}$, with $n$ being the angular quantum number. $\mathcal{R}_{i s n}\left(r / \ell_{c}\right)$ is the radial component of the wave function with confinement length $\ell_{c}$ - not to be confused with the topological charge-and radial quantum number $s$. The detailed functional form of $\mathcal{R}_{i s n}\left(r / \ell_{c}\right)$ depends on the confinement potential, which could be, e.g., square or parabolic (for the latter see Ref. [15] noticing a change in sign). The Bloch-periodic parts of electron states in the LH band are [27]

$$
\begin{aligned}
|3 / 2,+1 / 2\rangle & =-\frac{1}{\sqrt{6}}\left[\left(\left|p_{x}\right\rangle+i\left|p_{y}\right\rangle\right) \downarrow-2\left|p_{z}\right\rangle \uparrow\right], \\
|3 / 2,-1 / 2\rangle & =\frac{1}{\sqrt{6}}\left[\left(\left|p_{x}\right\rangle-i\left|p_{y}\right\rangle\right) \uparrow+2\left|p_{z}\right\rangle \downarrow\right] ;
\end{aligned}
$$

alternatively we will refer to these states as $u_{ \pm}(\mathbf{r})$. The $s$-like conduction-band states are written $u_{s}(\mathbf{r})=\langle\mathbf{r} \mid s\rangle \xi$, where $\xi$ is the spin.

In what follows, we will distinguish among different contributions to the angular momentum of carriers. For the sake of clarity we explicitly state them. Carriers have the band AM of their energy band: in the case of the $p$-like valence band this is equal to 1 , while for the $s$-type conduction band this is equal to 0 . In addition, electrons have spin AM equal to $1 / 2$. These two comprise the Bloch periodic-part or band+spin AM [Eq. (2)]. Finally, there is an envelope AM $\hbar n$ corresponding to the envelope part of the wave function.

\section{OPTICAL-VORTEX FIELD}

To be specific in what follows we use TL Bessel beams [12], which are one class of optical-vortex fields. However, much of our findings can be applied to other optical-vortex beams, as we explicitly discuss at the end of this section.

We study a monochromatic field of TL with wavelength $\lambda \simeq 500 \mathrm{~nm}$ and circular polarization $\sigma= \pm 1$ (polarization vector $\hat{x}+i \sigma \hat{y})$ propagating along the $\mathrm{QD}$ growth axis (i.e., impinging the sample at normal incidence). We use a nonparaxial expression for the vector potential [12]:

$$
\begin{aligned}
& A_{x}(\mathbf{r}, t)=F_{\ell}(r) \cos \left[\left(\omega t-q_{z} z\right)-\ell \varphi\right], \\
& A_{y}(\mathbf{r}, t)=\sigma F_{\ell}(r) \sin \left[\left(\omega t-q_{z} z\right)-\ell \varphi\right], \\
& A_{z}(\mathbf{r}, t)=-\sigma \frac{q_{r}}{q_{z}} F_{\ell+\sigma}(r) \sin \left[\left(\omega t-q_{z} z\right)-(\ell+\sigma) \varphi\right] .
\end{aligned}
$$

With $F_{\ell}(r)$ being a Bessel function, the vector potential satisfies the Coulomb gauge condition and the vectorial Helmholtz equation. Close to the phase singularity one can approximate $F_{\ell}(r) \simeq \xi A_{0}\left(q_{r} r\right)^{|\ell|} /|\ell|$ !, with $\xi=1\left[(-1)^{\ell}\right]$ for positive (negative) $\ell$. In the paraxial limit $\left(q_{r} / q_{z} \ll 1\right) A_{z}$ is negligible; then $\ell$ is the topological charge and the beam carries a single value of orbital AM. However, for a nonparaxial beam $\sigma$ and $\ell$ mix, and while $\left\{A_{x}, A_{y}\right\}$ retain their value $\ell$ for the topological charge, $A_{z}$ has a charge equal to $\ell+\sigma$. In what follows we will assume $\ell=+1$, but one can naturally choose $\ell=-1$, since the important distinction is that of the relative signs of $\sigma$ and $\ell$. Furthermore the beam is assumed to be highly focused such that $q_{r} / q_{z} \simeq 1$, where $\sqrt{q_{r}^{2}+q_{z}^{2}}=2 \pi / \lambda$, and $q_{r} \simeq 10^{-2} \mathrm{~nm}^{-1}$ is a measure of the beam waist.

Elsewhere [23] we have shown that a convenient mathematical representation of TL close to the phase singularity (located at $r=0$ ) is obtained by the so-called TL-gauge. This gauge transformation casts the Hamiltonian into an all-electric dipolar-like Hamiltonian when $\operatorname{sgn}(\ell)=\operatorname{sgn}(\sigma)$. For light with $\operatorname{sgn}(\ell) \neq \operatorname{sgn}(\sigma)$, magnetic terms appear that should not be $a$ priori neglected. Magnetic terms indeed overcome electric terms, for particular values of $\ell[21,23,24]$. However, for $\{\ell=+1, \sigma=-1\}$, the parameters chosen for the QD and beam, and the fact that we treat $\mathrm{LH}$ excitations, all magnetic contributions are negligible (see the Appendix) and we can indeed use the TL-gauge Hamiltonian also in this case. The direct consequence is that we can restrict our analysis to just electric interactions for both polarizations.

For light with topological charge $\ell=+1$ and polarization $\sigma=-1$ [using $E=-\partial_{t} A$ and Eq. (3) with $F_{\ell}(r) \simeq$ 
$\left.A_{0}\left(q_{r} r\right)^{\ell} / \ell !\right]$

$$
\begin{aligned}
& E_{x}(\mathbf{r}, t)=\frac{E_{0}}{2}\left(q_{r} r\right) \sin \left(\omega t-q_{z} z-\varphi\right), \\
& E_{y}(\mathbf{r}, t)=\frac{E_{0}}{2}\left(q_{r} r\right) \cos \left(\omega t-q_{z} z-\varphi\right), \\
& E_{z}(\mathbf{r}, t)=-E_{0} \frac{q_{r}}{q_{z}} \cos \left(\omega t-q_{z} z\right),
\end{aligned}
$$

with $E_{0}=\omega A_{0}$. The dominant term is the $z$ component, because the others are a factor $\left(q_{r} r\right)<0.1$ smaller. The Hamiltonian reads

$$
H=\frac{1}{2 m} \mathbf{p}^{2}+V(\mathbf{r})-q z E_{z}(\mathbf{r}, t),
$$

where $q$ is the electron charge, and, since magnetic terms can be neglected, the canonical momentum $\mathbf{p}$ is equal to the mechanical momentum.

For light with topological charge $\ell=+1$ and polarization $\sigma=+1$,

$$
\begin{aligned}
& E_{x}(\mathbf{r}, t)=\frac{E_{0}}{2}\left(q_{r} r\right) \sin \left(\omega t-q_{z} z-\varphi\right), \\
& E_{y}(\mathbf{r}, t)=-\frac{E_{0}}{2}\left(q_{r} r\right) \cos \left(\omega t-q_{z} z-\varphi\right), \\
& E_{z}(\mathbf{r}, t)=\frac{E_{0}}{8}\left(q_{r} r\right)^{2} \cos \left(\omega t-q_{z} z-2 \varphi\right),
\end{aligned}
$$

the in-plane components of the electric field dominate the interaction, with the Hamiltonian

$$
H=\frac{\mathbf{p}^{2}}{2 m}+V(\mathbf{r})-\frac{1}{2} q \mathbf{r}_{\perp} \cdot \mathbf{E}(\mathbf{r}, t),
$$

where $\mathbf{r}_{\perp}=x \hat{x}+y \hat{y}$. The extra factor $1 / 2$ is a result of the TL gauge and is related to the vanishing of the field at the phase singularity [23].

An interaction Hamiltonian such as $H_{I}=-q z E_{z}(\mathbf{r}, t)$ contains operators acting on the envelope and the periodic part of the wave function. This is clearly seen by writing $\mathbf{r}=\mathbf{R}+\mathbf{r}_{c}$, where $\mathbf{r}_{c}$ designates points within a crystal unit cell and $\mathbf{R}$ designates different unit cells in the whole crystal. The change of variable leads to $H_{I}=-q\left(\mathbf{R}+\mathbf{r}_{c}\right) \cdot \mathbf{E}\left(\mathbf{R}+\mathbf{r}_{c}\right)$. Since the electric field varies little within the unit cell we can disregard $\mathbf{r}_{c}$ in the argument of the electric field. The same cannot be done for the rest and indeed each term gives rise to different processes. Interband transitions are described by $H_{I}^{\text {inter }}=-q \mathbf{r}_{c} \cdot \mathbf{E}(\mathbf{R})$, while intraband transitions by $H_{I}^{\text {intra }}=$ $-q \mathbf{R} \cdot \mathbf{E}(\mathbf{R})$. We study in this article interband transitions.

To conclude the section, we briefly comment on the similar behavior exhibited by other optical-vortex fields. The focusing of a paraxial optical vortex by a lens has been shown to produce a conversion between orbital and spin AM [11], as it was commented after Eq. (3). Other authors have studied the specific case of focusing of an incoming Laguerre-Gaussian mode of TL with $\ell=1$ in experiments [22] and theory [20,21]. These works are in agreement with our nonparaxial electric field expressions Eqs. (4) and (6). In particular, they found that for $\sigma=-1$ only the $z$ component of the electric field survives at the phase singularity, while for $\sigma=1$ all components vanish. Calculations also show that the $r$ dependence of the field components is as in Eq. (4) for both polarization states of the light and small $q_{r} r$. In addition, radially/azimuthally polarized beams, which are superpositions of $\ell= \pm 1$ [28], exhibit similar features. For example, for focused radially polarized beams there is a nonvanishing $z$ component of the electric field at the phase singularity [29,30]. Besides, Biss et al. [31] have shown that radially/azimuthally polarized beams retain their spatial profile when focused through a dielectric interface, such as those of semiconductor heterostructures. This implies that the experimental control of the light hole in a QD can be realized by focusing either Bessel or Laguerre-Gaussian TL modes. Also, focused radially polarized beams can be used for the partial control of light holes, as proposed with $\{\ell=+1, \sigma=-1\}$ TL below.

\section{LIGHT-HOLE TRANSITIONS INDUCED BY AN OPTICAL VORTEX}

Unlike HHs, LHs are characterized by an admixture of different spin and band AM contributions, as seen in Eq. (2), that gives rise to richer optical transitions. The transitions create e-h pairs. For strong confinement potential in QDs [32] the Coulomb interaction, which accounts for exciton complexes, can be treated perturbatively [33]. Since we only consider small QDs, we describe optical transitions without the Coulomb interaction-new features related to the interaction of TL and excitons in bulk were studied in Refs. [16-18].

When a light beam with in-plane fields impinges on the QD, an e-h pair is created, due to the first term in the decomposition of $|3 / 2, \pm 1 / 2\rangle$ in Eq. (2). The pair thus produced has an AM projection equal to \pm 1 , due to the combination of conductionband electron and LH band+spin AM. Moreover, if the light carries no orbital $\mathrm{AM}(\ell=0)$, the transition is the one shown in Fig. 1 by the solid (black) line. In contrast, twisted light can transfer its orbital AM to the pair, by promoting an electron from an $s$-like envelope state in the valence band to a $p$-like envelope state in the conduction band as exemplified by the dashed (blue) line with $\ell=+1$.

The second term in the decomposition of hole states in Eq. (2) can only be addressed by a longitudinal $z$ component (parallel to the QD's growth axis) of the electric field. The

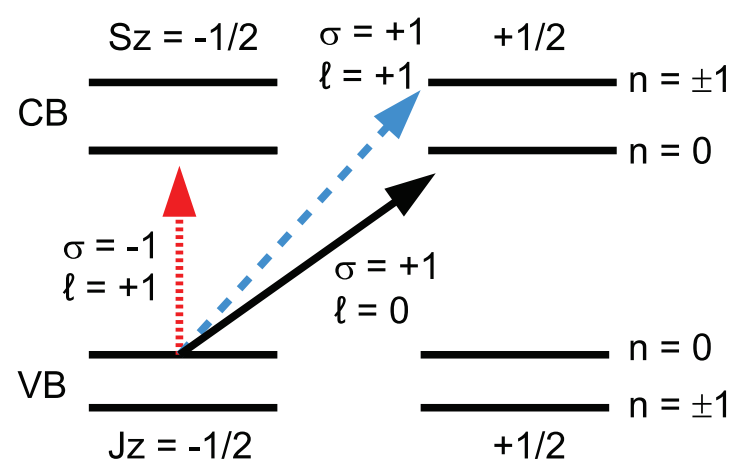

FIG. 1. (Color online) Transitions induced by light having $\ell=0,1$ on an electron initially in the $|3 / 2,-1 / 2\rangle$ LH band. The $z$ component of light with $\{\ell=+1, \sigma=-1\}$ produces an e-LH pair with $\mathrm{AM}=0$ [dotted (red)]. The in-plane component of light with $\sigma=+1$ produces an e-LH pair with AM $=1$ whose envelope AM is $n=0$ for $\ell=0$ [solid (black)] and $n=1$ for $\ell=+1$ [dashed (blue)]. 
resulting state is an e-h pair with $\mathrm{AM}=0$; see the dotted (red) lines in Fig. 1. Pairs with $\mathrm{AM}=0$ can be excited by plane-wave light propagating along the $x-y$ plane [6], although the corresponding experimental setup is usually cumbersome or even not possible.

In the next sections we will show that twisted light can produce both types of e-h pairs. This is done by calculating the transition matrix elements, which are essential to analyze excitation processes using for example Fermi's golden rule.

\section{A. Electron-light hole pairs with no band+spin angular momentum}

The excitation of an e-h pair with $\mathrm{AM}=0$ can be accomplished by twisted light having $\{\ell=+1, \sigma=-1\}$, thanks to its strong $z$-component electric field [see Eq. (4)]. We evaluate the matrix elements for the transition between $\mathrm{LH}$ and conduction bands. Under the assumption of nearly resonance excitation the rotating-wave approximation is used and the matrix element for the absorption of a photon, with $H_{I}=-q z_{c} E_{z}(Z, t)$, is

$$
\begin{aligned}
\left\langle\psi_{c}\left|H_{I}\right| \psi_{L H}\right\rangle \simeq & q \frac{E_{0}}{2} \frac{q_{r}}{q_{z}} e^{-i\left(\omega t-q_{z} Z\right)} \\
& \times\left\langle u_{s}\left|z_{c}\right| u_{ \pm}\right\rangle \delta_{s^{\prime} s} \delta_{n^{\prime} n},
\end{aligned}
$$

where both LHs are equally excited. Note that for a QD smaller than the beam waist $E_{z}$ depends neither on $r$ nor on $\varphi$. Moreover, since the QD is assumed quasi-2D, to a good approximation the $\exp \left(i q_{z} Z\right)$ can be pulled out of the matrix element. The consequence is that no new selection rules exist, as signalled by the delta functions in the envelope wave function quantum numbers. According to Eq. (2) $\left\langle u_{s}\left|z_{c}\right| u_{ \pm}\right\rangle=2 / \sqrt{6} z_{c v}$ with $z_{c v}=\left\langle s\left|z_{c}\right| p_{z}\right\rangle \simeq 1 \mathrm{~nm}$ $[27,34]$. Since the second term in the LH decomposition has entered the calculation and the spin is not modified by the light-matter interaction, we see that the excitation produces a hole $|3 / 2,+1 / 2\rangle(|3 / 2,-1 / 2\rangle)$ with a conduction-band electron with spin down (up); we remind the reader that holes have opposite quantum numbers to electrons. Then, the excitation obviously produces pairs of $\mathrm{AM}=0$. This is schematically represented in Fig. 1 by a dotted (red) line for the transition that promotes an electron from the state $|3 / 2,-1 / 2\rangle$.

\section{B. Electron-light hole pairs with angular momentum}

In Sec. IV A we have seen that the $z$ component of the EM field of a TL beam having $\{\ell=+1, \sigma=-1\}$ does not vanish at the phase singularity. This is a desirable feature, for it ensures a large fluency at the QD, and short exposure times would result in full transitions. However, we notice that this set of parameters renders a $z$ component of the field with no angular dependence [see Eq. (4)]. The features of nonvanishing amplitude at $r=0$ and lack of angular dependence coexist, in the case of the magnetic field, in another set of values $\{\ell=+2, \sigma=-1\}$ as one observes by inspection of the EM-field expressions deduced in other works [20-23]. Thus, fields having a finite amplitude at $r=0$ that would yield large fluency would not transfer orbital angular momentum to the material system. In contrast, the in-plane components of Eqs. (4) and (6) with vanishing amplitude at $r=0$ can indeed transfer orbital AM [to account for transitions induced by the in-plane field Eq. (4), one must add to the Hamiltonian Eq. (5) a new term proportional to $\left.q \mathbf{r}_{\perp} \cdot \mathbf{E}\right]$.

It is our aim in this section to show that TL can be used to address the remaining e-h pair states. By this we mean that e-h pairs having band + spin $A M=1$ can be created. Moreover, by the use of $\ell \neq 0$ e-h pairs with envelope AM are produced. One could consider the antiparallel AM fields of Sec. IV A, but the excitation is dominated by the $z$ component. We thus turn to the study of a beam having $\{\ell=+1, \sigma=+1\}$, in which case the $z$ component can be disregarded; other possible values of $\ell$ could be considered, and would produce transitions to other $n$ states, but the amplitude of the electric field close to the origin of coordinates diminishes with increasing orbital AM [15].

Following the discussion in Sec. IV A we calculate the matrix elements corresponding to the absorption of a photon in the rotating-wave approximation from the interaction Hamiltonian $(-1 / 2) q \mathbf{r}_{\perp} \cdot \mathbf{E}$ [Eq. (7)]

$$
\begin{aligned}
\left\langle\psi_{c s^{\prime} n^{\prime}}\left|H_{I}\right| \psi_{L H s n}\right\rangle= & -i \frac{q E_{0} x_{v c}}{4 \sqrt{6}} e^{-i\left(\omega t-q_{z} Z\right)} \\
& \times\left\langle s^{\prime} n^{\prime}\left|\left(q_{r} R\right) e^{i \varphi}\right| s n\right\rangle \delta_{L H,-1 / 2},
\end{aligned}
$$

where we used the separation of the matrix element into Bloch-periodic and envelope parts, and the decomposition of LHs in $p$-like orbitals Eq. (2). As in Eq. (8) $x_{v c} \simeq 1 \mathrm{~nm}$. Since we have considered only the case where the polarization of the field is $\sigma=1$, there is actually one possible transition, the one originating from $|3 / 2,-1 / 2\rangle$ as signalled by the delta function. Using Eq. (1) the envelope-function matrix element is

$$
\begin{aligned}
\left\langle s^{\prime} n^{\prime}\left|\left(q_{r} R\right) e^{i \varphi}\right| s n\right\rangle= & \delta_{n+1, n^{\prime}} \int_{0}^{\infty} d R\left(q_{r} R^{2}\right) \\
& \times \mathcal{R}_{i s^{\prime} n+1}^{*}(R) \mathcal{R}_{i s n}(R) .
\end{aligned}
$$

Of main interest is the transition from $\{s=0, n\}$ to $\{s=0$, $(n+1)\}$. For a parabolic confinement potential with radial Laguerre-Gaussian wave functions, the integral has the analytical solution $(\sqrt{2} \pi)^{-1} q_{r} \ell_{c} \sqrt{n+1}$. The product $q_{r} \ell_{c}$ makes explicit the importance of a well-focused beam. Twisted light can be effectively focused using an objective, so reducing the beam waist such that $q_{r} \simeq q_{z}$ [20]. In this case the transition with $n=0$ (uppermost valence-band state) yields $\left\langle 01\left|\left(q_{r} R\right) e^{i \varphi}\right| 00\right\rangle \simeq 3 \times 10^{-2}$. As already discussed, the vanishing of the field at $r=0$ reduces the probability amplitude [15] making necessary the use of longer and/or more intense pulses to complete a transition.

A different strategy to reduce the exposure time and intensity of the field is to use semiconductor systems with larger $E_{g}$. For example, QDs based on GaN/AIN are excited at $q_{z} \simeq 300 \mathrm{~nm}^{-1}$, so improving the factor $q_{r} \ell_{c}$. They confine both electrons and holes and can be modeled by Eq. (1) with Bessel radial wave functions.

\section{SPINTRONIC APPLICATIONS}

In this section we will show how a single-beam configuration setup using one or multiple pulses of an optical-vortex field at normal incidence can be used to improve previous 
proposals using LH exciton dynamics in single QDs, or can be used in new ways.

\section{A. Controlling magnetic impurities}

In the past years several groups have been successful in placing a single magnetic ion, such as $\mathrm{Mn}$ or Co, in a single QD of either II-VI or III-V material [35-37]. The spin of such a magnetic impurity interacts with the electrons and holes in the QD; this interaction is usually well described by Heisenbergtype couplings between the various spins involved [38,39]. This interaction gives rise to a characteristic splitting of the exciton line of the QD into a multiplet of lines, the details being strongly dependent on both the QD material and the impurity atom [35-39].

The spin degree of freedom of a Mn impurity in a II-VI QD has has been proposed for applications in the field of spintronics or quantum information processing due to its long lifetime [40]. For this purpose it is necessary to selectively prepare the Mn spin in any of its six possible orientations. Various schemes have been proposed to achieve this task by coherent manipulation of excitons $[6,41,42]$. These schemes are based on combined spin flips of electron and Mn spin or hole and $\mathrm{Mn}$ spin. In the case of $\mathrm{HH}$ excitons, only electron spin flips are possible because a Heisenberg-type Hamiltonian cannot produce transitions from a state with $m_{j}=+3 / 2$ to $m_{j}=-3 / 2$ or vice versa. In contrast, in the case of LH excitons both types of spin flips are possible, which makes this type of exciton particularly attractive to achieve the Mn spin preparation.

The basic idea behind the Mn spin switching schemes is the following: By optical excitation with a given polarization an exciton with a certain AM is created. Then, a correlated dynamics of the Mn and the exciton spin system starts and at a suitable later time when the exciton is in a state with a different AM, this exciton is removed again by a light pulse with the respective polarization. Restricting oneself to circularly polarized plane-wave beams, only Mn spin flips by multiples of two are possible, because only excitons with AM \pm 1 can be created or removed. However, by adding linearly polarized light with polarization along the $z$ direction also LH excitons with AM 0 can be created or removed and thus the Mn spin can be driven into all of its six eigenstates [6]. Using only plane-wave light, this scheme however requires the application of light pulses propagating in normal incidence direction and in an in-plane direction. From a practical point of view, such a double-beam setup is cumbersome, because it requires light propagating in the QD plane and therefore typically cleaving of the sample is needed. We propose here the use of a single normal-incidence beam having either the values $\{\ell=0, \sigma= \pm 1\}$ to address LH excitons with $\mathrm{AM} \pm 1$, or $\{\ell= \pm 1, \sigma=\mp 1\}$ to address excitons with AM 0. Switching between one and the other value of light's orbital AM can be achieved by the use of two collinear beams (a plane-wave one and the other of TL) or by switching the topological charge of a single beam [43].

In addition, twisted light offers a way to control the relative strength of the interaction between carriers and impurities. This interaction is typically well described by a contact interaction and its strength is therefore proportional to the modulus square of the wave function of the involved carrier at the impurity position [44]. Electrons and holes are usually excited by plane waves, producing electron-hole pairs where both carriers have the same orbital AM; e.g., $n=0$ in the $s$ shell or $n=1$ in the $p$ shell. Thus, both carriers have their largest spatial probability either close to the QD center ( $s$-shell states) or away from the center ( $p$-shell states). Regardless of the impurity position-which varies from sample to sample due to fabrication uncontrollable parameters - the ratio between the electron-impurity and the hole-impurity interaction will be essentially the same in all samples.

Consider instead the excitation by TL with $\{\ell=+1$, $\sigma=+1\}$ as depicted by dashed (blue) line in Fig. 1. The electron and hole are created in the envelope $p(n=1)$ and $s(n=0)$ states, respectively. Likewise, by tuning the light frequency to the appropriate transition, an electron-hole pair with electron in the envelope $s(n=0)$ and hole in the envelope $p(n=1)$ state can be created. Thus, for an impurity placed at the center, in the former case the interaction with the hole will be strongly dominant while in the latter case the electronimpurity interaction will dominate. Conversely, if the impurity is at some distance from the QD center, in the first case the electron and in the second case the hole will interact stronger. Magnetic fields can be used to further tune the transition frequency and therefore select which carrier (electron or hole) is excited into an orbital state with $n \neq 0$ [15].

One can even imagine the use of superposition of twisted light beams having different $\ell$ to further narrow the localization region of carriers. Consider for example the simultaneous excitation with an $\{\ell=+1, \sigma=+1\}$ and an $(\ell=-1$, $\sigma=+1)$ beam. If the laser frequency is tuned to the transition between the valence-band state $n=0$ and the conduction-band state $n=1$, the in-plane components of both fields will be responsible for the interaction. Then, an electron in a superposition state of envelope $n=+1$ and $n=-1$ with spin up will be generated. By introducing a relative phase between the two beams, the spatial orientation of the electron's wave function can be rotated, thus allowing to better control the interaction strengths with an impurity located away from the QD center.

\section{B. Controlling the spin of an excess electron}

Let us now consider the control of the spin of an excess electron in a charged QD by employing the $z$ component of the TL field. Pazy et al. [7,45] have suggested the use of a combination of two pulses traveling in orthogonal directions to achieve such a spin flip. As was previously mentioned, this idea faces implementation problems. For example, a realistic quantum computing proposal based on semiconductor technology would use a 2D array of a large number of charged QDs [3], where each excess electron realizes a qubit, and the spin-flip implements a Pauli-X gate. For this proposal, the use of light beams propagating in the plane of the array seems difficult or even impossible. However, as in other situations [19], the $z$ component of the optical vortex can prove very handy by producing the spin flip at normal incidence.

Relying on recent experiments [46], we propose the use of optical-vortex pulses $P_{\ell, \sigma}$ in the tens of femtoseconds range. Note that the pulse duration is mainly limited by 


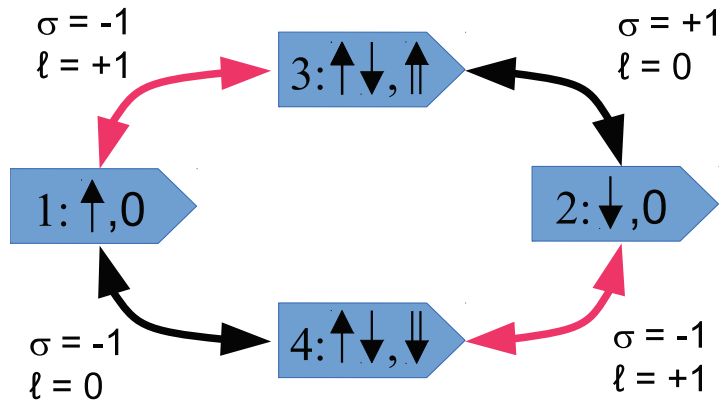

FIG. 2. (Color online) Control of an excess electron in a charged QD, states and possible transitions. Single arrows indicate the spin of electrons in the conduction band; double arrows and zero indicate the AM projection of LHs in the valence band.

the spectral selectivity required for a specific sample. A sequence of normal-incidence $\pi$ pulses, $\left\{P_{1,-1} \rightarrow P_{0,1} \rightarrow\right.$ $\left.P_{0,-1}\right\}$ (first pulse left), excites the charged QD at normal incidence. The relevant states are the single-electron states $\{|1\rangle=|\uparrow, 0\rangle,|2\rangle=|\downarrow, 0\rangle\}$ and the charged exciton (trion) states $\{|3\rangle=|\uparrow \downarrow, \uparrow\rangle,|4\rangle=|\uparrow \downarrow, \downarrow\rangle\}$, where the arrows $\uparrow, \downarrow$ refer to the electron spin and $\Uparrow, \Downarrow$ and zero to the LH AM. Figure 2 shows a graphical representation of the states and possible transitions. Such a pulse sequence can easily be seen to produce spin inversion of the excess electron. For ultrashort excitation, the whole evolution of the system can be broken into intervals of free evolution and pulse excitation [47]. We will denote by $U_{\ell, \sigma}$ the evolution operator for a certain time interval in which an optical-vortex pulse $P_{\ell, \sigma}$ with pulse area $\pi$ is present. Let us assume that the initial state of the system is $|1\rangle$. Applying to it the aforementioned sequence of pulses, we get the final state

$$
\begin{aligned}
\left|\psi_{f}\right\rangle & =U_{0,-1} U_{0,1} U_{1,-1}|1\rangle \\
& =U_{0,-1} U_{0,1}|3\rangle=U_{0,-1}|2\rangle=|2\rangle,
\end{aligned}
$$

disregarding the global phase arising from free evolution. On the other hand, starting with the initial state $|2\rangle$, we get

$$
\begin{aligned}
\left|\psi_{f}\right\rangle & =U_{0,-1} U_{0,1} U_{1,-1}|2\rangle \\
& =U_{0,-1} U_{0,1}|4\rangle=U_{0,-1}|4\rangle=|1\rangle .
\end{aligned}
$$

Thus, in both cases we obtain an inversion of the electron spin. We note that if the initial state is known, two pulses are sufficient, as in the first case the third pulse has no action on the state and in the second case this holds for the second pulse. The three-pulse sequence, however, works in both cases and it also works for an arbitrary superposition of the two electron spin states, as we see by a numerical simulation of the density matrix $\rho(t)$ using the master-equation formalism within the 4-level system. Figure 3 shows the evolution starting from a pure superposition state $|\Psi\rangle\langle\Psi|$ with $|\Psi\rangle=a_{1}|1\rangle+a_{2}|2\rangle$. A full spin inversion, equivalent to a Pauli- $X$ gate, is completed in less than a picosecond using moderate laser powers $\left(E_{0} \simeq 10^{9} \mathrm{~V} / \mathrm{m}\right)$. It is worth noting that there was no need to include decay and dephasing processes. While the excitation and deexcitation of e-h pairs takes place in our protocol in less than a ps, the e-h recombination and dephasing times are in the hundreds of ps $[48,49]$. In addition, the spin dephasing time of conduction-band electron
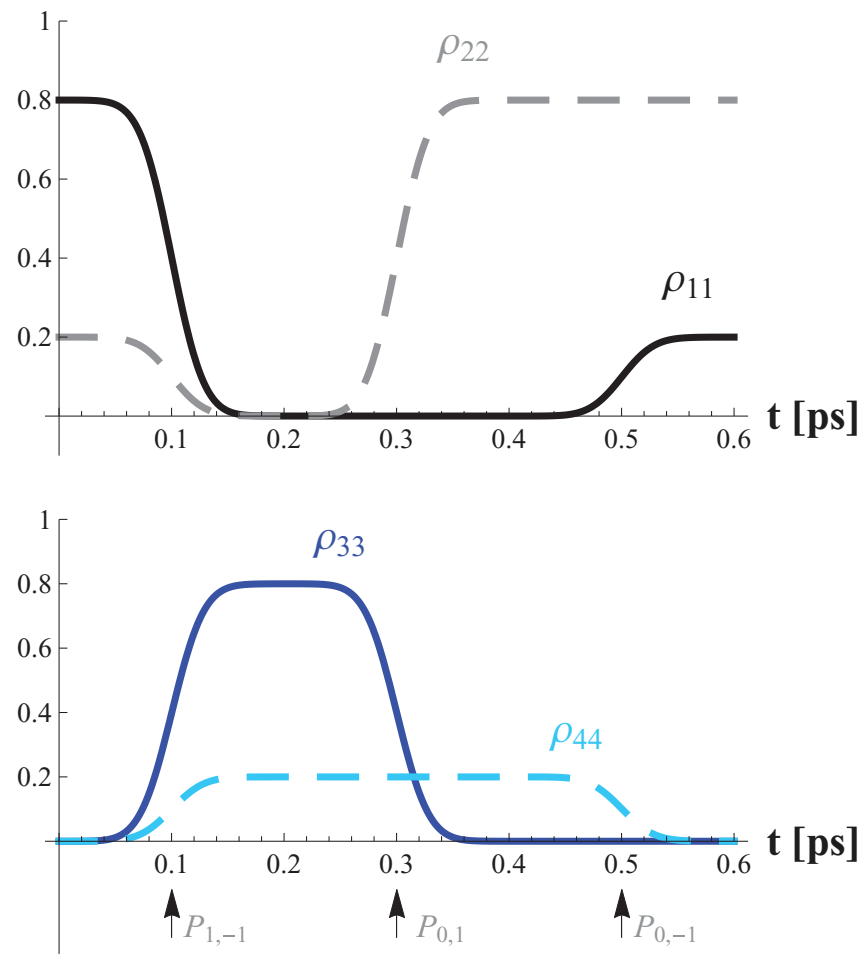

FIG. 3. (Color online) Evolution of the density matrix from an initial pure state with $a_{1}^{2}=0.8$ and $a_{2}^{2}=0.2$. The sequence of ultrashort $\pi$ pulses $\left\{P_{1,-1}, P_{0,1}, P_{0,-1}\right\}$ is applied at times $t=0.1,0.3,0.5 \mathrm{ps}$, respectively; each Gaussian pulse lasts for $\tau_{p}=50 \mathrm{fs}$.

is in the nano- to microsecond range [3,5,50]. However, some other factors are detrimental to the fidelity of the operation, for instance the detuning of the laser and deviations of the pulse area from the correct value $\pi$. This sensitivity to pulse parameters might be overcome by employing the technique of adiabatic rapid passage using chirped pulses [51-53].

Due to the short operation time, one could even relax the requirement of energy inversion between light and heavy hole bands, and so the present proposal would be compatible with QDs where the HH is the lowest hole state.

\section{EXCITATION OF SINGLE AND MULTIPLE QUANTUM DOTS}

In order to support future experimental testing of the above ideas, we briefly comment on the topic of positioning of the beam and the selective excitation and measurement of systems with a single QD as well as an extension to samples with multiple QDs. For the interaction of QDs with an optical vortex this is a delicate issue, as was discussed in Ref. [54]. To analyze the situation, we have to distinguish between two modes of excitation, namely the one using the $z$ component of the light field and the other aiming at a transfer of orbital angular momentum.

The $z$ component of the electric field in the case of a beam with $\{\ell=+1, \sigma=-1\}$ has its maximum at the beam center located at $r=0$ and varies smoothly and slowly in the neighborhood of this point [the correction to the constant field 
in $r=0$ is of order $\left.\left(q_{r} r\right)^{2}\right]$. Thus, there is no need to precisely center the beam axis on top of a given QD. Furthermore, this insensitivity with respect to the exact positioning may even be employed to extend our scheme to a selective excitation of different QDs in a sample consisting of an ensemble of QDs. In such samples, there are usually several QDs in the area illuminated by the light beam (with a radius of a fraction of a micrometer). Since these QDs are essentially never identical they can be selectively addressed in the spectral range, in much the same way as is usually done for excitation by nonvortex light. Typical densities of QDs on samples grown for single-QD spectroscopy are in the range of $10^{9}-10^{10} \mathrm{~cm}^{-2}$ $[55,56]$, which means that there are on average a few up to a few tens of QDs in the range of the beam; all of them will have slightly different geometries and therefore different excitation frequencies [55]. In addition, by growing QDs on a patterned substrate, QDs with different spectral features can even be positioned in a controlled way allowing for selective single-dot excitation [57,58]. Also, vertically stacked QDs have been fabricated [59] which can be selectively addressed as long as the barrier width is sufficiently large such that they behave like individual dots [60].

On the other hand, we have elsewhere shown that the selective transfer of orbital angular momentum to the wave function of carriers requires that the symmetry axes of QD and beam coincide [54]. Then, the positioning of the beam or sample must be accurate on the order of a fraction of the QD size, which can be achieved by the use of current-technology techniques and equipment, e.g., high-precision positioning stages. QDs that are displaced from the beam axis would also be excited-if they were located close to the maximum of the field profile the excitation might even be much strongerhowever, they would not present a clear selection rule for the transfer of OAM. Therefore, when applying this scheme to an ensemble of laterally displaced QDs, a precise positioning on top of a single QD is necessary and by selecting the appropriate frequency only this QD should be excited. To excite another QD at a different position a new positioning of the beam would be required. However, vertically stacked QD are usually well aligned among themselves. This implies that in this case a single multicolor beam with suitably adjusted frequencies might address multiple QDs still preserving the selection rule for the OAM.

\section{CONCLUSIONS}

We have theoretically studied the excitation of light holes in small quantum dots by highly focused optical-vortex beams. To this end we employed the twisted-light gauge which casts the interaction Hamiltonian in terms of electric fields, and can account for the simultaneous action of all three spatial components that typically appear in tightly focused beams.

It was shown that a single optical-vortex beam at normal incidence can create all possible microscopic states of electronhole pairs in the quantum dot, and we gave explicit expressions for the corresponding transition matrix elements.

Then, we applied our results to present proposals in spintronics, namely the manipulation of the spin of a magnetic impurity or of an excess electron in quantum dots, and demonstrated that those proposals can be improved by using optical-vortex fields. We also suggested a new possible way to address states of magnetic impurities, based on the transfer of orbital angular momentum to the envelope function of carriers in the quantum dot.

Finally we discussed the important issue of beam positioning and selective excitation and measurement of single- and multiple-QD systems. The discussion is meant to support and facilitate future experimental testing of the ideas presented in the article.

\section{ACKNOWLEDGMENT}

G.F.Q. thanks the Argentine research agency CONICET for financial support through the "Programa de Becas Externas".

\section{APPENDIX: NEGLECTING MAGNETIC TERMS}

In Ref. [23] we have shown that a gauge transformation of the type

$$
\begin{aligned}
\chi(\mathbf{r}, t)= & -\frac{1}{|\ell|+1} \mathbf{r}_{\perp} \cdot \mathbf{A}(\mathbf{r}, t) \\
& -\frac{1}{|\ell+\sigma|+1} z A_{z}(\mathbf{r}, t)
\end{aligned}
$$

leads to a convenient representation of the interaction Hamiltonian. For light having $\operatorname{sgn}(\sigma)=\operatorname{sgn}(\ell)$, the Hamiltonian is given solely in terms of electric fields, while for $\operatorname{sgn}(\sigma)$ $\neq \operatorname{sgn}(\ell)$, the vector potential after performing the gauge transformation is not necessarily negligible. Therefore, in the latter case magnetic contributions, i.e., contributions resulting from the remaining $\mathbf{p} \cdot \mathbf{A}$ coupling, should in principle be taken into account.

We will next show that, for $\{\ell=+1, \sigma=-1\}$ with the chosen parameters for QD and beam, the magnetic terms are in fact negligible when one considers LH to conduction-band transitions. The gauge transformation applied to Eq. (3) produces a new scalar potential

$$
U^{\prime}(\mathbf{r}, t)=-\frac{1}{2} \mathbf{r}_{\perp} \cdot \mathbf{E}(\mathbf{r}, t)-z E_{z}(\mathbf{r}, t)
$$

and a new vector potential

$$
\begin{aligned}
& A_{\varphi}^{\prime}=\frac{A_{0}}{2}\left(q_{r} r\right) \sin (\Phi), \\
& A_{z}^{\prime}=A_{0} \frac{q_{r}}{q_{z}}\left(q_{z} z\right) \cos (\Phi)-\frac{A_{0}}{4} \frac{q_{z}}{q_{r}}\left(q_{r} r\right)^{2} \sin (\Phi),
\end{aligned}
$$

with $\Phi=\omega t-q_{z} z$. In Sec. III we have seen that the dominant component of the electric field is $E_{z}$ [see Eq. (4)]. Therefore, we are interested in comparing the magnetic interactions corresponding to Eq. (A3) with the $z$-component part of the electric interaction.

As an example, we provide details on the relative strength of magnetic and electric interactions arising from the $z$ components. We calculate the ratio of interband matrix element $\left\langle q\left(p_{z} / m\right) A_{z}^{\prime}\right\rangle$ to $\left\langle q z E_{z}\right\rangle$. In the former $A_{z}^{\prime}$ acts on the envelope part of the wave function, while $p_{z}$ acts on the Bloch-periodic part; thus, one can split the matrix element into $\left\langle u_{c}\left|q\left(p_{z} / m\right)\right| u_{v}\right\rangle\left\langle\phi_{f} \mathcal{Z}_{f}\left|A_{z}^{\prime}\right| \phi_{i} \mathcal{Z}_{i}\right\rangle$. Furthermore, one can apply the well-known relationship $\left\langle u_{c}\left|p_{z}\right| u_{v}\right\rangle=$ $-i m \omega_{c v}\left\langle u_{c}|z| u_{v}\right\rangle$. On the other hand, $E_{z}$ can be pulled out of its matrix element, since it does not depend on coordinates. We 
note that the magnetic and electric terms may connect different initial and final states, and they should all be compared. Let us next consider each term from $A_{z}^{\prime}$ separately:

$$
\begin{aligned}
\frac{\left\langle q\left(p_{z} / m\right) A_{z 1}^{\prime}\right\rangle}{\left\langle q z E_{z}\right\rangle} & =\omega_{c v} \frac{\left\langle\phi_{f} \mathcal{Z}_{f}\left|A_{z 1}^{\prime}\right| \phi_{i} \mathcal{Z}_{i}\right\rangle}{E_{z}} \\
& =\frac{q_{r}}{q_{z}} \frac{\omega_{c v}}{\omega}\left\langle\phi_{i} \mathcal{Z}_{f}\left|\left(q_{z} z\right)\right| \phi_{i} \mathcal{Z}_{i}\right\rangle
\end{aligned}
$$

The operator $z$ forces initial and final states to be of opposite parity. Since the QD is quasi-2D, the next $z$ subband has a much larger energy, and thus is well detuned from the transitions considered in the present paper. Moreover, the matrix element is proportional to $\left(q_{z} z\right) \ll 1$. Thus, it is safe to neglect the first term of $A_{z}^{\prime}$. Next, we look at the second term

$$
\begin{aligned}
\frac{\left\langle q\left(p_{z} / m\right) A_{z 2}^{\prime}\right\rangle}{\left\langle q z E_{z}\right\rangle} & =\omega_{c v} \frac{\left\langle\phi_{f} \mathcal{Z}_{f}\left|A_{z 2}^{\prime}\right| \phi_{i} \mathcal{Z}_{i}\right\rangle}{E_{z}} \\
& =\frac{1}{4} \frac{q_{z}}{q_{r}} \frac{\omega_{c v}}{\omega}\left\langle\phi_{f} \mathcal{Z}_{i}\left|\left(q_{r} r\right)^{2}\right| \phi_{i} \mathcal{Z}_{i}\right\rangle .
\end{aligned}
$$

Since the $|\phi \mathcal{Z}\rangle$ are not eigenstates of the operator $r$, we can only argue that the ratio is proportional to $\left(q_{r} r\right)^{2} \ll 1$, and thus is also negligible. In conclusion, we see that the magnetic field interaction is much smaller than the electric interaction considered in the article.

Similar arguments can be given for $(q / m) p_{\varphi} A_{\varphi}$ and $(-1 / 2) \mathbf{d}_{\perp} \cdot$ E. As a result, the Hamiltonian reads

$$
H=\frac{1}{2 m} \mathbf{p}^{2}+V(\mathbf{r})-d_{z} E_{z}(\mathbf{r}, t) .
$$

If instead of LH-CB transitions, $\mathrm{HH}-\mathrm{CB}$ transitions are studied, the interaction terms arising from the in-plane components of $\mathbf{A}$ and $\mathbf{E}$ must be compared. According to Eq. (4) and Eq. (A3) these are proportional to $\left(q_{r} r\right)$; therefore, the ratio of the magnetic to electric interactions is a constant, and one cannot disregard magnetic effects.
[1] E. M. Gauger, S. C. Benjamin, A. Nazir, and B. W. Lovett, Phys. Rev. B 77, 115322 (2008).

[2] H. Jayakumar, A. Predojević, T. Huber, T. Kauten, G. S. Solomon, and G. Weihs, Phys. Rev. Lett. 110, 135505 (2013).

[3] K. De Greve, D. Press, P. L. McMahon, and Y. Yamamoto, Rep. Prog. Phys. 76, 092501 (2013).

[4] P. M. Koenraad and M. E. Flatté, Nat. Mater. 10, 91 (2011).

[5] M. Feng, I. D'Amico, P. Zanardi, and F. Rossi, Europhys. Lett. 66, 14 (2004).

[6] D. Reiter, T. Kuhn, and V. Axt, Phys. Rev. B 83, 155322 (2011).

[7] E. Pazy, E. Biolatti, T. Calarco, I. D'amico, P. Zanardi, F. Rossi, and P. Zoller, Europhys. Lett. 62, 175 (2003).

[8] Y. H. Huo, B. J. Witek, S. Kumar, J. R. Cardenas, J. X. Zhang, N. Akopian, R. Singh, E. Zallo, R. Grifone, D. Kriegner et al., Nat. Phys. 10, 46 (2014).

[9] D. L. Andrews, Structured Light and Its Applications (Academic Press, London, 2008).

[10] S. M. Barnett and L. Allen, Opt. Commun. 110, 670 (1994).

[11] K. Y. Bliokh, E. A. Ostrovskaya, M. A. Alonso, O. G. RodríguezHerrera, D. Lara, and C. Dainty, Opt. Express 19, 26132 (2011).

[12] G. F. Quinteiro and P. I. Tamborenea, Europhys. Lett. 85, 47001 (2009).

[13] G. F. Quinteiro and J. Berakdar, Opt. Express 17, 20465 (2009).

[14] J. Wätzel, A. S. Moskalenko, and J. Berakdar, Opt. Express 20, 27792 (2012).

[15] G. F. Quinteiro and P. I. Tamborenea, Phys. Rev. B 79, 155450 (2009).

[16] Y. Ueno, Y. Toda, S. Adachi, R. Morita, and T. Tawara, Opt. Express 17, 20567 (2009).

[17] G. F. Quinteiro, Europhys. Lett. 91, 27002 (2010).

[18] K. Shigematsu, Y. Toda, K. Yamane, and R. Morita, Jpn. J. Appl. Phys. 52, 08JL08 (2013).

[19] B. Sbierski, G. Quinteiro, and P. Tamborenea, J. Phys.: Condens. Matter 25, 385301 (2013).

[20] P. B. Monteiro, P. A. M. Neto, and H. M. Nussenzveig, Phys. Rev. A 79, 033830 (2009).

[21] V. V. Klimov, D. Bloch, M. Ducloy, and J. R. Rios Leite, Phys. Rev. A 85, 053834 (2012).
[22] Y. Iketaki, T. Watanabe, N. Bokor, and M. Fujii, Opt. Lett. 32, 2357 (2007).

[23] G. F. Quinteiro, D. E. Reiter, and T. Kuhn, arXiv:1401.8147.

[24] J. R. Zurita-Sánchez and L. Novotny, J. Opt. Soc. Am. B 19, 2722 (2002).

[25] S. Kako, K. Hoshino, S. Iwamoto, S. Ishida, and Y. Arakawa, Appl. Phys. Lett. 85, 64 (2004).

[26] A. Andreev and E. O'Reilly, Phys. Rev. B 62, 15851 (2000).

[27] G. Bastard, Wave Mechanics Applied to Semiconductor Heterostructures (Les Editions de Physique, Hasteld Press, France, 1988), Chap. VII.

[28] M. Ornigotti and A. Aiello, Opt. Express 21, 15530 (2013).

[29] R. Dorn, S. Quabis, and G. Leuchs, Phys. Rev. Lett. 91, 233901 (2003).

[30] K. Youngworth and T. Brown, Opt. Express 7, 77 (2000).

[31] D. Biss and T. Brown, Opt. Express 9, 490 (2001).

[32] A. B. Dzyubenko and A. Y. Sivachenko, J. de Physique IV 3, C5-381 (1993).

[33] A. Wojs and P. Hawrylak, Phys. Rev. B 51, 10880 (1995).

[34] P. Sergey, J. Nanostruct. Chem. 3, 1 (2013).

[35] L. Besombes, Y. Léger, L. Maingault, D. Ferrand, H. Mariette, and J. Cibert, Phys. Rev. Lett. 93, 207403 (2004).

[36] A. Kudelski, A. Lemaître, A. Miard, P. Voisin, T. Graham, R. J. Warburton, and O. Krebs, Phys. Rev. Lett. 99, 247209 (2007).

[37] J. Kobak, T. Smoleński, M. Goryca, M. Papaj, K. Gietka, A. Bogucki, M. Koperski, J.-G. Rousset, J. Suffczyński, E. Janik et al., Nat. Commun. 5, 3191 (2014).

[38] J. Fernández-Rossier, Phys. Rev. B 73, 045301 (2006).

[39] J. van Bree, P. M. Koenraad, and J. Fernández-Rossier, Phys. Rev. B 78, 165414 (2008).

[40] C. Le Gall, R. S. Kolodka, C. L. Cao, H. Boukari, H. Mariette, J. Fernández-Rossier, and L. Besombes, Phys. Rev. B 81, 245315 (2010).

[41] D. E. Reiter, T. Kuhn, and V. M. Axt, Phys. Rev. Lett. 102, 177403 (2009).

[42] D. E. Reiter, T. Kuhn, and V. M. Axt, Phys. Rev. B 85, 045308 (2012). 
[43] M. Mirhosseini, O. S. Magana-Loaiza, C. Chen, B. Rodenburg, M. Malik, and R. W. Boyd, Opt. Express 21, 30196 (2013).

[44] A. H. Trojnar, M. Korkusiński, M. Potemski, and P. Hawrylak, Phys. Rev. B 85, 165415 (2012).

[45] T. Calarco, A. Datta, P. Fedichev, E. Pazy, and P. Zoller, Phys. Rev. A 68, 012310 (2003).

[46] K. Yamane, Y. Toda, and R. Morita, Opt. Express 20, 18986 (2012).

[47] L. Viola and S. Lloyd, Phys. Rev. A 58, 2733 (1998).

[48] A. Melliti, M. Maaref, F. Hassen, M. Hjiri, H. Maaref, J. Tignon, and B. Sermage, Solid State Commun. 128, 213 (2003).

[49] X. Mu, Y. J. Ding, B. S. Ooi, and M. Hopkinson, in Conference on Lasers and Electro-Optics (Optical Society of America, Washington DC, 2007), p. JThD69.

[50] A. Greilich, D. R. Yakovlev, and M. Bayer, Solid State Commun. 149, 1466 (2009).

[51] Y. Wu, I. M. Piper, M. Ediger, P. Brereton, E. Schmidgall, P. R. Eastham, M. Hugues, M. Hopkinson, and R. T. Phillips, Phys. Rev. Lett. 106, 067401 (2011).

[52] C.-M. Simon, T. Belhadj, B. Chatel, T. Amand, P. Renucci, A. Lemaitre, O. Krebs, P. Dalgarno, R. Warburton, X. Marie et al., Phys. Rev. Lett. 106, 166801 (2011).
[53] S. Lüker, K. Gawarecki, D. E. Reiter, A. Grodecka-Grad, V. M. Axt, P. Machnikowski, and T. Kuhn, Phys. Rev. B 85, 121302(R) (2012).

[54] G. Quinteiro, A. Lucero, and P. Tamborenea, J. Phys.: Condens. Matter 22, 505802 (2010).

[55] T. Makino, R. Andre, J. Gerard, R. Romestain, L. S. Dang, M. Bartels, K. Lischka, and D. Schikora, Appl. Phys. Lett. 82 2227 (2003).

[56] T. Kazimierczuk, J. Suffczyński, A. Golnik, J. A. Gaj, P. Kossacki, and P. Wojnar, Phys. Rev. B 79, 153301 (2009).

[57] D. Dalacu, K. Mnaymneh, V. Sazonova, P. J. Poole, G. C. Aers, R. Cheriton, M. Reimer, J. Lapointe, P. Hawrylak, M. Korkusinski et al., Proc. SPIE 7608, 76082G (2010).

[58] D. Dalacu, M. E. Reimer, S. Frederick, D. Kim, J. Lapointe, P. J. Poole, G. C. Aers, R. L. Williams, W. Ross McKinnon, M. Korkusinski et al., Laser Photon. Rev. 4, 283 (2010).

[59] D. Bruls, P. Koenraad, H. Salemink, J. Wolter, M. Hopkinson, and M. Skolnick, Appl. Phys. Lett. 82, 3758 (2003).

[60] C. A. Kessler, M. Reischle, R. Roßbach, E. Koroknay, M. Jetter, H. Schweizer, and P. Michler, Phys. Status Solidi (b) 249, 747 (2012). 